\title{
Changes in gastric microbiota induced by Helicobacter pylori infection and preventive effects of Lactobacillus plantarum ZDY 2013 against such infection
}

\author{
Mingfang Pan, ${ }^{\star} \dagger$ Cuixiang Wan, $\uparrow$ Qiong Xie, $†$ Renhui Huang, $†$ Xueying Tao, $†$ Nagendra $P$. Shah, ${ }^{*} \ddagger^{1}$ \\ and Hua Wei* $\dagger^{1}$ \\ ${ }^{*}$ College of Life Science, Nanchang University, Nanchang 330031, P. R. China \\ †State Key Laboratory of Food Science and Technology, Nanchang University, Nanchang, Jiangxi 330047, P. R. China \\ †Food and Nutritional Science, School of Biological Sciences, University of Hong Kong, Hong Kong
}

\begin{abstract}
Helicobacter pylori is a gram-negative pathogen linked to gastric ulcers and stomach cancer. Gastric microbiota might play an essential role in the pathogenesis of these stomach diseases. In this study, we investigated the preventive effect of a probiotic candidate Lactobacillus plantarum ZDY 2013 as a protective agent against the gastric mucosal inflammation and alteration of gastric microbiota induced by $H$. pylori infection in a mouse model. Prior to infection, mice were pretreated with or without $400 \mu \mathrm{L}$ of L. plantarum ZDY 2013 at a concentration of $10^{9} \mathrm{cfu} / \mathrm{mL}$ per mouse. At 6 wk postinfection, gastric mucosal immune response and alteration in gastric microbiota mice were examined by quantitative real-time PCR and high-throughput $16 \mathrm{~S}$ rRNA gene amplicon sequencing, respectively. The results showed that L. plantarum ZDY 2013 pretreatment prevented increase in inflammatory cytokines (e.g., IL-1 $\beta$ and IFN- $\gamma$ ) and inflammatory cell infiltration in gastric lamina propria induced by $H$. pylori infection. Weighted UniFrac principal coordinate analysis showed that L. plantarum ZDY 2013 pretreatment prevented the alteration in gastric microbiota post- H. pylori infection. Linear discriminant analysis coupled with effect size identified 22 bacterial taxa (e.g., Pasteurellaceae, Erysipelotrichaceae, Halomonadaceae, Helicobacteraceae, and Spirochaetaceae) that overgrew in the gastric microbiota of $H$. pylori-infected mice, and most of them belonged to the Proteobacteria phylum. Lactobacillus plantarum ZDY 2013 pretreatment prevented this alteration; only 6 taxa (e.g., Lachnospiraceae, Ruminococcaceae, and Clostridiaceae), mainly from the taxa of Firmicutes and Bacteroidetes, were dominant in the gastric microbiota of the L. plantarum ZDY 2013 pretreated mice. Administration of L. plantarum
\end{abstract}

Received October 10, 2015.

Accepted November 6, 2015.

${ }^{1}$ Corresponding authors: weihua@ncu.edu.cn and npshah@hku.hk
ZDY 2013 for $3 \mathrm{wk}$ led to increase in several bacterial taxa (e.g., Rikenella, Staphylococcus, Bifidobacterium), although a nonsignificant alteration was found in the gastric microbiota. Overall, this study demonstrated that L. plantarum ZDY 2013 pretreatment played an important role in preventing gastric mucosal inflammation and gastric microbiota alteration induced by $H$. pylori infection, and the selective modulation in gastric microbiota posed by this intervention suggested that targeting gastric microbiota through oral administration of probiotics might be an alternative strategy to prevent $H$. pylori infection.

Key words: gastric microbiota, Helicobacter pylori, probiotic, Lactobacillus plantarum ZDY 2013, highthroughput

\section{INTRODUCTION}

Helicobacter pylori is a highly prevalent pathogen that colonizes approximately half of the world's population (Polk and Peek, 2010; Wroblewski et al., 2010). Although most subjects colonized by $H$. pylori are asymptomatic, this pathogen is strongly associated with gastritis, peptic ulcers, and gastric cancer in humans (Salama et al., 2013). A complex disease is rarely caused by a single factor. Previous studies have identified several factors that determine the disease outcome post- $H$. pylori infection (Martin and Solnick, 2014), whereas most of them have focused on the virulence factors carried by H. pylori, host genetics, and environment. Recently, with a rapid improvement and availability of sequencing-based analysis techniques, the role of host microbiota has attracted the interest of scientists to study disease associated with H. pylori infection (Sheh and Fox, 2013; Yang et al., 2013).

Several studies have indicated that microbiota is involved in shaping host physiology and balance of immune system (Clemente et al., 2012), and altered microbiota is strongly associated with several diseases and conditions such as inflammatory bowel disease (Packey 
and Sartor, 2008), obesity (Ley et al., 2005), diabetes (Xu et al., 2015), and even liver cirrhosis (Qin et al., 2014). Previous studies have demonstrated that gastric microbiota played an important role in the development of gastric cancer (Wang et al., 2014). In particular, a recent study has demonstrated a gradual shift in the gastric microbiota profile from nonatrophic gastritis to intestinal metaplasia and then to intestinal-type gastric cancer (Aviles-Jimenez et al., 2014), indicating that an interaction took place between gastric microbiota and H. pylori. However, whether a specific pre-infection microbiota of the host would affect colonization of $H$. pylori and subsequent outcome after intervention with this microorganism for gastric disease is not yet known.

Probiotics are defined as "live microorganisms which, when administered in adequate amounts, may have a positive effect to alter the microflora of host and exert a health benefit to the host" (Joint FAO/WHO Working Group, 2002). Oral administration of probiotic bacteria has been used as an alternative strategy to combat several gastrointestinal diseases (e.g., acute infectious diarrhea; Weichert et al., 2012). Currently, the role of probiotics in the treatment of H. pylori-related gastrointestinal diseases is increasingly being recognized as an alternate or complement to antibiotics, with a potential to decrease the use of antibiotics or to reduce their side effects (Patel et al., 2014). Several studies have shown that some probiotic strains (Saccharomyces boulardii, Lactobacillus acidophilus, or mixtures of strains) have specific mechanisms against $H$. pylori infection, including inhibiting $H$. pylori attachment to mucosal cells (Sakarya and Gunay, 2014), reducing H. pyloriassociated gastric inflammation (Yang and Sheu, 2012), or other direct physiological effects (Gotteland et al., 2006). A recent meta-analysis found that adjunct use of a few probiotic strains improved H. pylori eradication rates and prevented the development of adverse events and antibiotic-associated diarrhea in those treated with standard eradication therapies (McFarland et al., 2015). Despite these advances, effects of probiotics on gastric microbiota, and a relationship between those effects and the disease outcome caused by H. pylori infection, are not yet examined.

In the present study, L. plantarum ZDY 2013, isolated from traditional Chinese fermented soybeans, was selected as probiotic as it exhibited antimicrobial properties in vitro (Pan et al., 2015) and showed modulatory effects on gut microbiota in mice (Huang et al., 2015). Pretreatment of C57BL/6J mice with this microorganism was carried out before $H$. pylori infection, and gastric mucosal immune response and changes in gastric microbiota post- $H$. pylori infection were examined by using quantitative real-time PCR (qPCR) assay and high-throughput 16S rRNA gene amplicon sequencing, respectively. Our results showed that pretreatment with L. plantarum ZDY 2013 prevented gastric inflammation and changes in microbiota post- $H$. pylori infection. Moreover, 3 wk L. plantarum ZDY 2013 administration induced selective modulation in gastric microbiota, suggesting targeting the gastric microbiota through probiotics may be a new avenue for preventing $H$. pylori infection.

\section{MATERIALS AND METHODS}

\section{Bacterial Strain and Growth Condition}

Lactobacillus plantarum ZDY 2013 was grown in de Man, Rogosa, Sharpe (MRS broth; Beijing Solarbio Science \& Technology Co. Ltd., Beijing, China) under anaerobic condition at $37^{\circ} \mathrm{C}$ overnight when the viable cell population reached at approximately $10^{9} \mathrm{cfu} / \mathrm{mL}$ for administration to mice.

The Sydney strain of Helicobacter pylori (SS1) was cultured on Campylobacter agar base (Oxoid, Basingstoke, UK) supplemented with 5\% defibrinated horse blood (Solarbio, Beijing, China), $10 \mu \mathrm{g} / \mathrm{mL}$ vancomycin, 2.5 units $/ \mathrm{mL}$ polymyxin $\mathrm{B}, 5 \mu \mathrm{g} / \mathrm{mL}$ amphotericin, and $5 \mu \mathrm{g} / \mathrm{mL}$ trimethoprim under micro-aerophilic conditions $\left(5 \% \mathrm{O}_{2}, 10 \% \mathrm{CO}_{2}\right.$, and $\left.85 \% \mathrm{~N}_{2}\right)$ at $37^{\circ} \mathrm{C}$ for $48 \mathrm{~h}$. For mice infection, H. pylori was grown in Brain Heart Infusion (Oxoid) supplemented with $10 \%$ fetal bovine serum (ExCell Bio, Shanghai, China) with gentle shaking at $37^{\circ} \mathrm{C}$ under micro-aerophilic conditions overnight.

\section{Animal Experiments}

The protocol for carrying out animal experiments was approved by Nanchang University Animal Ethics Committee. All the animal experiments were approved by the Animal Care Review Committee (approval number 0064257) of Nanchang University (Jiangxi, China). Female specific-pathogen-free C57BL/6J mice (SJA Laboratory Animal Co. Ltd., Hunan, China) were housed at the animal facility under standard conditions in sterile cages with sterile bedding and supplemented with sterile feed and water ad libitum. The timeline for the animal experiment is depicted in Figure 1A. Briefly, after 1 wk of acclimatization, 32 mice ( 16 g, 6 wk old) were randomly divided into 4 groups receiving 4 different pretreatments as described below:

- Group NC (n=8): normal chow diet with $400 \mu \mathrm{L}$ of PBS (control),

- Group LP $(\mathrm{n}=8)$ : normal chow diet with $400 \mu \mathrm{L}$ of viable cells of L. plantarum ZDY $2013\left(\sim 10^{9}\right.$ 
$\mathrm{cfu} / \mathrm{mL}$ ) suspended in PBS and administered by oral gavages,

- Group HP $(\mathrm{n}=8)$ : normal chow diet with 400 $\mu \mathrm{L}$ of PBS,

- Group LP+HP $(\mathrm{n}=8)$ : normal chow diet with $400 \mu \mathrm{L}$ of viable cells of L. plantarum ZDY 2013 $\left(\sim 10^{9} \mathrm{cfu} / \mathrm{mL}\right)$ suspended in PBS and administered by oral gavages.

After 3 wk of L. plantarum ZDY 2013 administration, mice in groups of HP and LP $+\mathrm{HP}$ were infected by oral gavages with $500 \mu \mathrm{L}$ of $H$. pylori $\left(\sim 10^{8} \mathrm{cfu} / \mathrm{mL}\right)$ per mouse twice at a 1-d interval. The mice were fasted overnight before gavages. The animals were killed via $\mathrm{CO}_{2}$ narcosis at $6 \mathrm{wk}$ postinfection; the stomachs were dissected and opened along the lesser curvature. After washing in cold sterile normal saline, the stomachs were divided into longitudinal strips and fixed in paraformaldehyde for hematoxylin-eosin staining or immediately frozen in liquid nitrogen before stored in $-80^{\circ} \mathrm{C}$ for DNA and RNA extraction.

\section{Stomach Histology}

Paraformaldehyde-fixed stomach tissues were embedded in paraffin, sectioned at $5 \mathrm{~mm}$, and then stained with hematoxylin and eosin as previously described (Zhang et al., 2013).

\section{qPCR Assay}

Quantitative analysis of IL- $1 \beta$, IFN- $\gamma$, IL-17, and IL-10 in the mouse stomach tissues was performed by qPCR. Total RNA was isolated from the tissue samples of the mouse stomach using Takara MiniBEST Universal RNA Extraction Kit according to the manufacturer's protocol. The RNA (500 ng) was reverse transcribed into cDNA using the Takara PrimeScript RT reagent

A

Pre-treatment with H.pylori

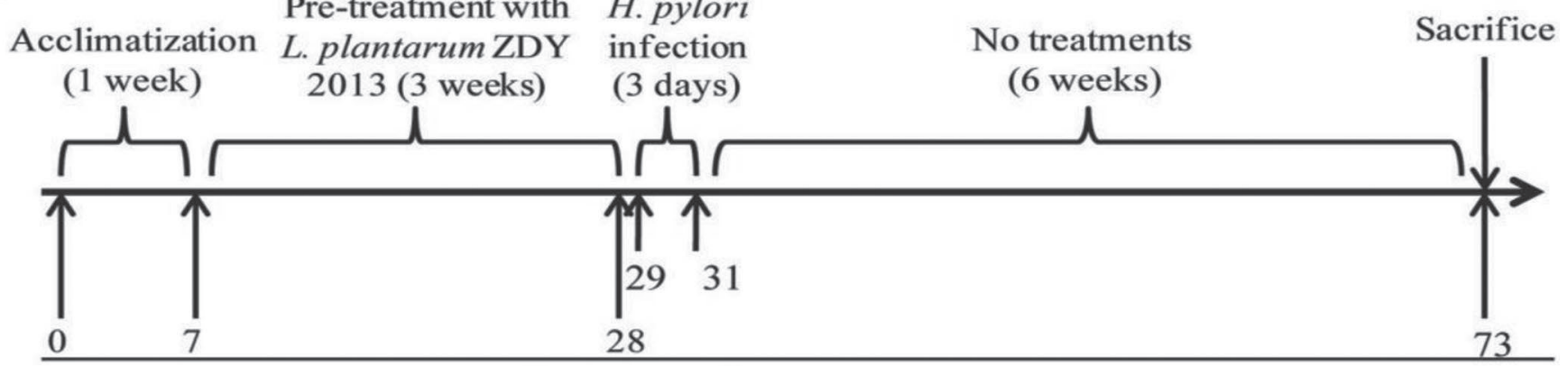

Time point (day)

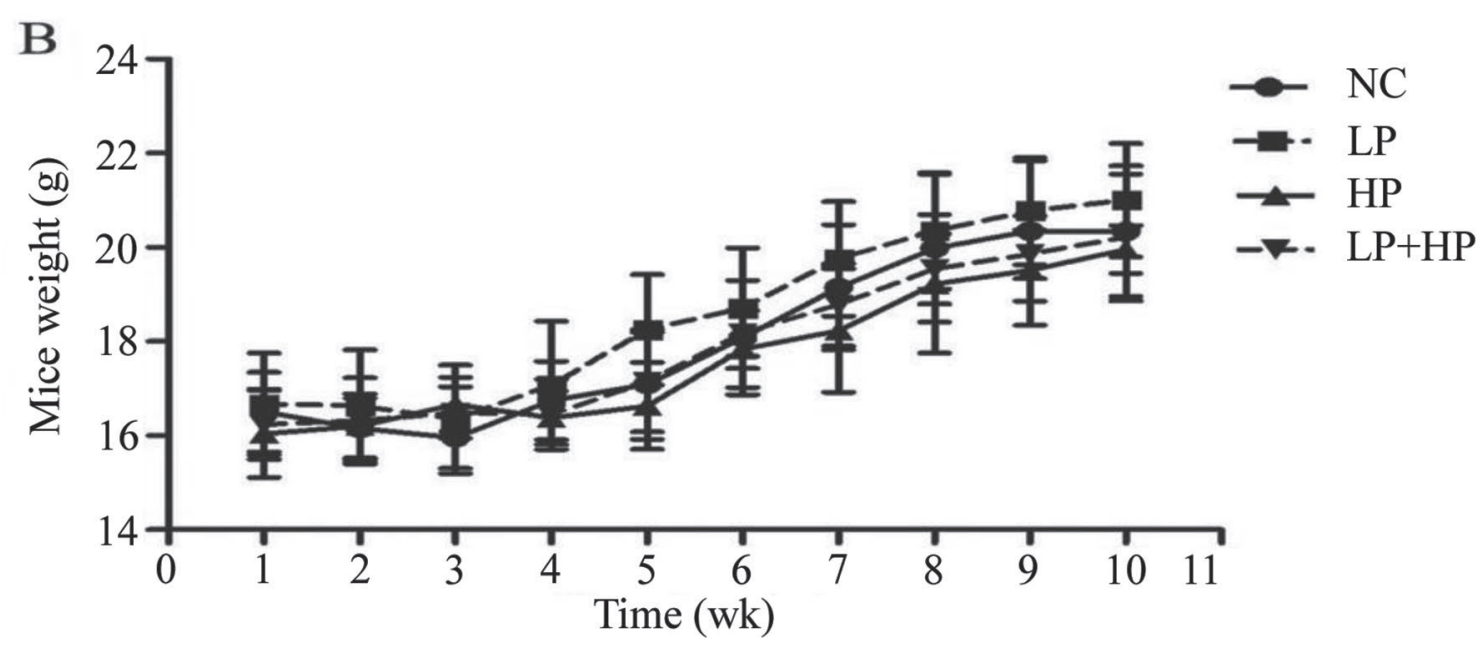

Figure 1. (A) Timeline for animal experiment. (B) Body weight of mice in the 4 groups. NC $=$ normal chow diet with $400 \mu \mathrm{L}$ of PBS (control); LP $=$ normal chow diet with $400 \mu \mathrm{L}$ of viable cells of Lactobacillus plantarum ZDY $2013\left(\sim 10^{9} \mathrm{cfu} / \mathrm{mL}\right)$ suspended in PBS and administered by oral gavages; $\mathrm{HP}=$ normal chow diet with $400 \mu \mathrm{L}$ of PBS; LP $+\mathrm{HP}=$ normal chow diet with $400 \mu \mathrm{L}$ of viable cells of $L$. plantarum ZDY $2013\left(\sim 10^{9} \mathrm{cfu} / \mathrm{mL}\right)$ suspended in PBS and administered by oral gavages. Error bars represent mean \pm standard deviation. 
Table 1. Primer sequences for mouse inflammatory cytokine genes

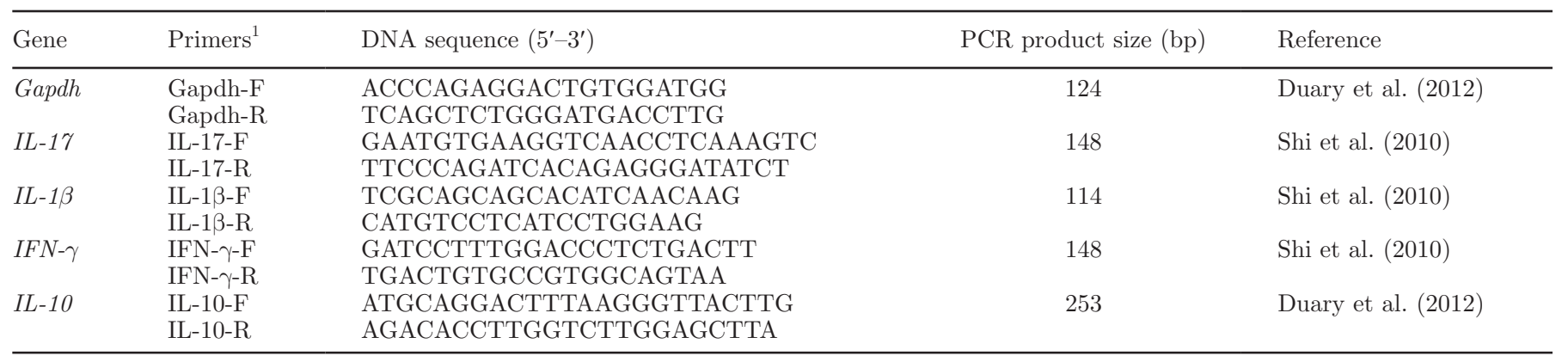

${ }^{1} \mathrm{~F}=$ forward; $\mathrm{R}=$ reverse.

Kit with gDNA Erase. The qPCR was performed using SYBR Premix Ex Taq II. Primers were synthesized by Invitrogen China (Shanghai, China), and are listed in Table 1. Glyceraldehyde-3-phosphate dehydrogenase (Gadph) was used as the housekeeping gene. The 20$\mu \mathrm{L}$ reaction mix contained $0.8 \mu \mathrm{L}(10 \mu M)$ of forward and reverse primers, $0.4 \mu \mathrm{L}$ of ROX Reference Dye II $(50 \times), 10 \mu \mathrm{L}$ of SYBR Premix Ex Taq II $(2 \times), 2 \mu \mathrm{L}$ of cDNA samples, and the rest with nuclease-free water. The assays were performed on the 7900HT Fast RealTime System (Applied Biosystems, Foster City, CA) with the following thermocycling program: pre-amplification stage $95^{\circ} \mathrm{C}$ for $1 \mathrm{~min}$ and then 40 cycles at $95^{\circ} \mathrm{C}$ for $5 \mathrm{~s}$ and $60^{\circ} \mathrm{C}$ for $1 \mathrm{~min}$. All mRNA quantification data were normalized to the Gadph and are presented as fold change over control using the critical threshold (Ct) number and calculated using the $2^{-\Delta \Delta \mathrm{Ct}}$ method (Yang et al., 2015).

\section{Total DNA Extraction and 16S rRNA Gene Amplicon Sequencing}

Total genomic DNA was extracted from the gastric samples by using DNeasy Blood and Tissue Kit (Qiagen) combined with the bead-beater method (Bik et al., 2006). Briefly, gastric samples ( 30 mg) were lysed in $180 \mu \mathrm{L}$ of QIAamp ATL buffer and $20 \mu \mathrm{L}$ of proteinase $\mathrm{K}$ for $1 \mathrm{~h}$ at $56^{\circ} \mathrm{C}, 0.2 \mathrm{~g}$ of zirconium beads $(0.1 \mathrm{~mm})$ were added, and samples were homogenized by bead beating twice for $45 \mathrm{~s}$ at a setting of 5.0 using a FastPrep-24 System (MP Biomedicals, Santa Ana, $\mathrm{CA})$. An aliquot of $4 \mu \mathrm{L}$ of RNaseA $(100 \mathrm{mg} / \mathrm{mL})$ was added and incubated for $2 \mathrm{~min}$ at room temperature $\left(\sim 22^{\circ} \mathrm{C}\right)$. Then DNA was recovered according to the manufacturer's protocol for tissue samples. The DNA concentrations and purity were examined by agarose gel electrophoresis and spectrophotometry using the NanoDrop (Thermo Scientific, Wilmington, DE). Purified DNA was stored at $-80^{\circ} \mathrm{C}$ before $16 \mathrm{~S}$ rRNA gene amplicon sequencing.
The 16S rRNA gene amplicon sequencing was performed on the Illumina MiSeq platform (BGI Tech Solutions Co. Ltd., Shenzhen, China) according to protocols described by Caporaso et al. (2012). The sequences of the primers targeting the V4 hyper-variable region of the bacterial 16S rRNA genes were as follows: $515 \mathrm{~F}$, 50-GTGCCAGCMGCCGCGGTAA-30; and 806R, 50-GGACTACHVGGGTWTCTAAT-30.

\section{Bioinformatics Analysis}

Bioinformatic analysis of sequencing data was conducted using the quantitative insights into microbial ecology (QIIME) pipeline (Caporaso et al., 2010). Briefly, raw data from all samples were filtered to eliminate the adapter pollution and low quality to obtain clean reads. High quality paired-end reads were combined to tags with an average read length of 252 bp using FLASH (fast length adjustment of short reads, v. 1.2.11). The tags were then clustered to OTU using USEARCH (v. 7.0.1090) at $97 \%$ sequence similarity and into phylotypes by clustering all the sequences belonging to the same genus. Taxonomic ranks were assigned to OTU representative sequence using Ribosomal Database Project Classifier v 2.2. Finally, an operational taxonomic units (OTU) table and a phylogenetic tree were produced for further $\alpha$ diversity (within a sample) and $\beta$ diversity (between samples) analysis. Alpha diversity analysis included rarefaction curve, Shannon diversity index, and Simpson diversity. Principal coordinate analysis based on weighted or unweighted UniFrac was used for $\beta$ diversity analysis. The statistical significance of the microbiota separation between groups was assessed by Adonis test utilizing the distance matrix between groups directly in $\mathrm{R}$ (version 2.15; The R Project for Statistical Computing) with Vegan packages (Oksanen et al., 2013). Differentially abundant bacterial taxa between groups were compared by using linear discriminant analysis effect size (LEfSe) method (Segata et al., 2011). A significant $\alpha$ 
of 0.05 and an effect size threshold of 2 were used for all biomarkers discussed in this study.

\section{Statistical Analysis}

Data are expressed as mean \pm standard deviation. Statistical significant difference among the groups was determined by one-way ANOVA followed by Tukey's post hoc test. An unpaired 2-tailed Student's $t$-test was used to analyze the significant differences between 2 groups. A $P$-value $<0.05$ was considered statistically significant. GraphPad Prism version 5.01 for Windows (GraphPad Software Inc., La Jolla, CA) was used for statistical analysis.

\section{RESULTS}

\section{Effects of L. plantarum ZDY 2013 Pretreatment on Gastric Mucosal Inflammation}

Compared with the normal group, mice in the HP group showed a slight reduction in BW (Figure 1B) after wk 5 (1 wk postinfection) but exhibited a significantly elevated level $(P<0.01)$ of inflammatory cytokine mRNA for IL-1 $\beta$ (Figure $2 \mathrm{~A}$ ), IFN- $\gamma$ (Figure $2 \mathrm{~B}$ ), and IL-17 (Figure 2C) in gastric mucosa at $6 \mathrm{wk}$ postinfection. Lactobacillus plantarum ZDY 2013 pretreatment for 3 wk prevented the increase in mRNA for IL-1 $\beta$ (Figure 2A) and IFN- $\gamma$ (Figure 2B), and significantly reduced $(P<0.05)$ the mRNA level for IL-17 (Figure 2C). The mRNA level of IL-10 (Figure 2D) showed no significant changes among all the groups. Moreover, hematoxylin-eosin staining of the gastric mucosal tissue showed severe inflammatory cell infiltration in the gastric lamina propria of mice from HP group, whereas mice from LP $+\mathrm{HP}$ showed no inflammation (Figure 2E).

\section{Effects of L. plantarum ZDY 2013 Pretreatment on Gastric Bacterial Diversity and Bacterial Community Alteration}

Rarefaction curve (Figure 3A), Shannon's diversity index, and Simpson's diversity index (Figure 3B) were calculated to analyze the $\alpha$ diversity changes in gastric microbiota of the 4 groups (Figure 3A). In comparison, H. pylori infection led to a decrease in $\alpha$ diversity of gastric microbiota at 6 wk postinfection. Pretreatment with L. plantarum ZDY 2013 prevented the decrease in Shannon's diversity index and Simpson's diversity index (Figure 3B).

The changes in gastric microbiota across groups were examined by using principal coordinate analysis (PCoA) based on the unweighted and weighted Uni- frac distance matrixes. The microbiota in each group showed an apparent clustering based on unweighted distance matrixes (Figure 3C), but robust clustering based on weighted Unifrac distance matrixes (Figure 3D). While using the Adonis test, both of them yielded a $P$-value of 0.001 , indicating at least one group being separated from the others. To further analyze the effect of H. pylori infection and L. plantarum ZDY 2013 pretreatment on the gastric microbiota, a similar PCoA based on weighted Unifrac distance was carried out to compare the NC versus $\mathrm{HP}$ or $\mathrm{LP}+\mathrm{HP}$, and the Adonis test showed a significant difference in microbiota between NC and HP $(P=0.024)$, but not between NC and $\mathrm{LP}+\mathrm{HP}(P=0.084)$. Additionally, a nonsignificant microbiota difference was observed between NC and LP $(P=0.105$, Adonis test $)$.

\section{The Bacterial Taxa Response to H. pylori Infection and L. plantarum ZDY 2013 Pretreatment}

The gastric bacterial community composition of the 4 groups is shown in Figure 4. It was demonstrated that 4 phyla (in descending order: Firmicutes, Bacteroidetes, Proteobacteria, and Verrucomicrobia) were the most dominant in 13 bacterial phyla; Firmicutes and Bacteroidetes represented on an average as much as $75.6 \%$ of each sample's OTU.

To identify the specific bacterial taxa associated with H. pylori infection and L. plantarum ZDY 2013 pretreatment, the gastric microbiota in the group of HP was compared with that of $\mathrm{LP}+\mathrm{HP}$ using LEfSe. The greatest difference in taxa between the 2 communities is displayed in Figure 5A. Twenty-two bacterial taxa (e.g., Pasteurellaceae, Erysipelotrichaceae, Halomonadaceae, Helicobacteraceae, and Spirochaetaceae) were significantly $(P<0.05)$ much more abundant in HP group, whereas only 6 taxa were overgrown $(P<$ 0.05) in the group of LP+HP (e.g., Lachnospiraceae, Ruminococcaceae, and Clostridiaceae). Particularly, the average relative abundance of bacterial genus of Helicobacter in HP group was 0.0281, whereas it decreased to 0.006 in the LP+HP group (Figure 5B).

\section{Bacterial Taxa Associated with Administration for 3 wk of L. plantarum ZDY 2013}

The hypothesis that L. plantarum ZDY 2013 administration may change the gastric microbiota, which can pose a preventive effect against $H$. pylori infection, was interesting. To explore the bacteria taxa associated with L. plantarum ZDY 2013 administration, a LEfSe comparison of the gastric microbiota between the group $\mathrm{NC}$ and LP was performed. As shown in Figure 6, at family level, 5 bacterial families (i.e., Rikenellaceae, 
A
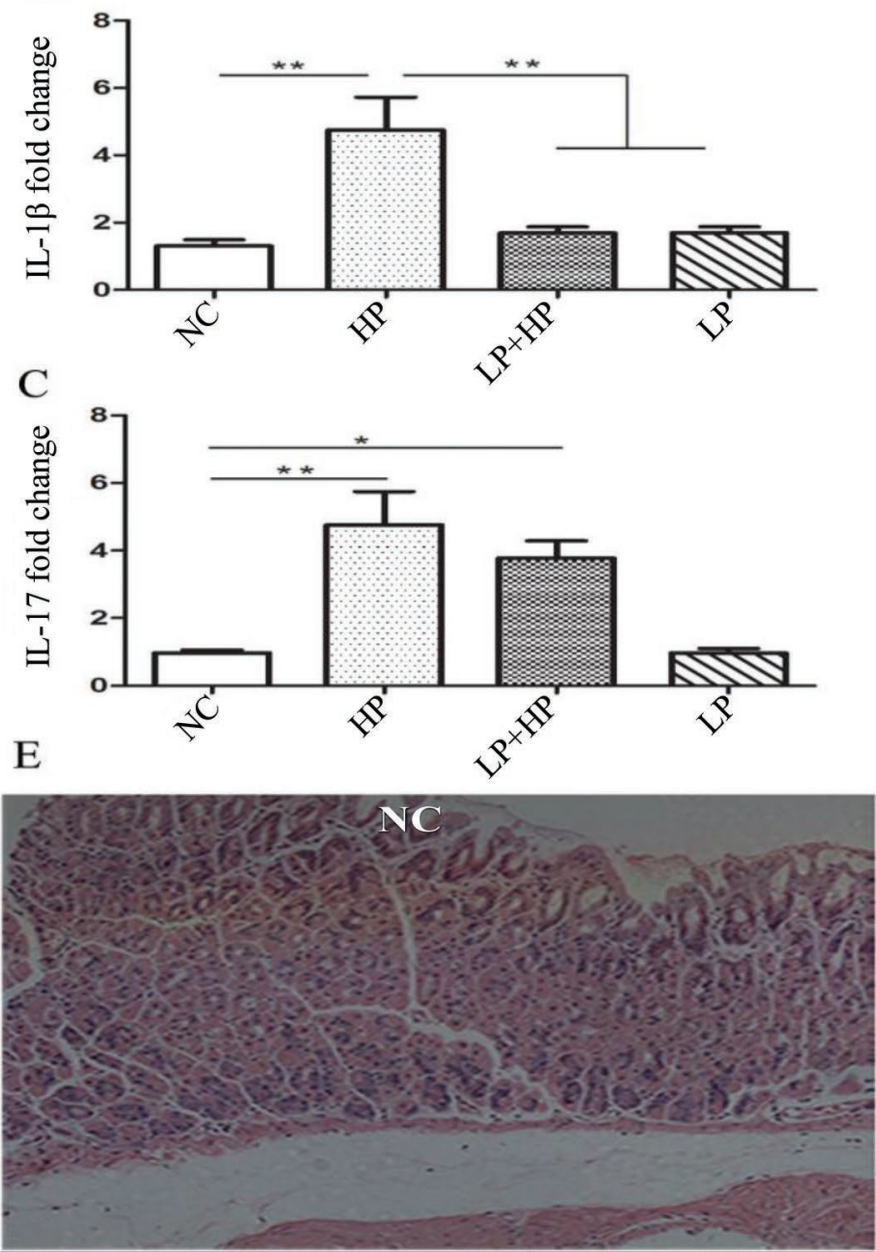

\section{LP}

\section{B}
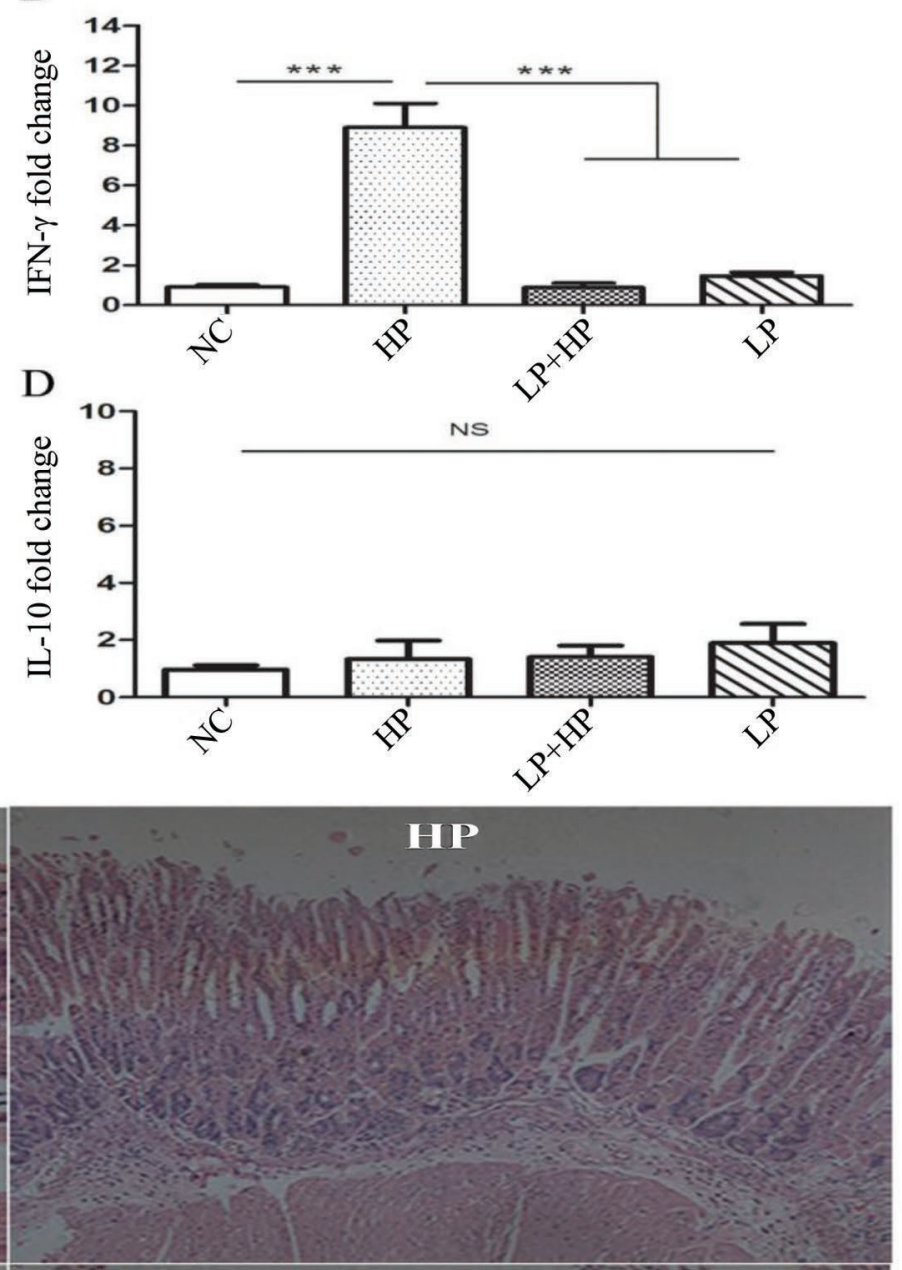

$\mathbf{L P}+\mathrm{HIP}$

Figure 2. The LP pretreatment suppresses gastric mucosal inflammation induced by Helicobacter pylori infection. Gastric mucosal inflammatory cytokine mRNA for IL-1 $\beta$ (A), IFN- $\gamma(\mathrm{B})$, and IL-17 (C) were significantly suppressed by LP pretreatment. Cytokine mRNA for IL-10 (D) showed no change. ${ }^{*} P<0.05 ;{ }^{*} P<0.01 ;{ }^{* * *} P<0.001$ by ANOVA followed by Tukey post hoc test. (E) Gastric histopathology from C57BL/6J mouse inoculated with $H$. pylori showed moderate inflammatory cell infiltration of the lamina propria, whereas 3 wk pretreatment of LP prevented inflammatory cell infiltration in lamina propria. Hematoxylin-eosin stain: original magnification $\times 150$. NC $=$ normal chow diet with $400 \mu \mathrm{L}$ of PBS (control); LP = normal chow diet with $400 \mu \mathrm{L}$ of viable cells of Lactobacillus plantarum ZDY 2013 ( $\sim 10^{9}$ cfu/ $\mathrm{mL}$ ) suspended in PBS and administered by oral gavages; HP = normal chow diet with $400 \mu \mathrm{L}$ of $\mathrm{PBS}$; LP $+\mathrm{HP}=$ normal chow diet with 400 $\mu \mathrm{L}$ of viable cells of $L$. plantarum ZDY $2013\left(\sim 10^{9} \mathrm{cfu} / \mathrm{mL}\right)$ suspended in PBS and administered by oral gavages. Error bars represent mean \pm standard deviation. Color version available online. 
A
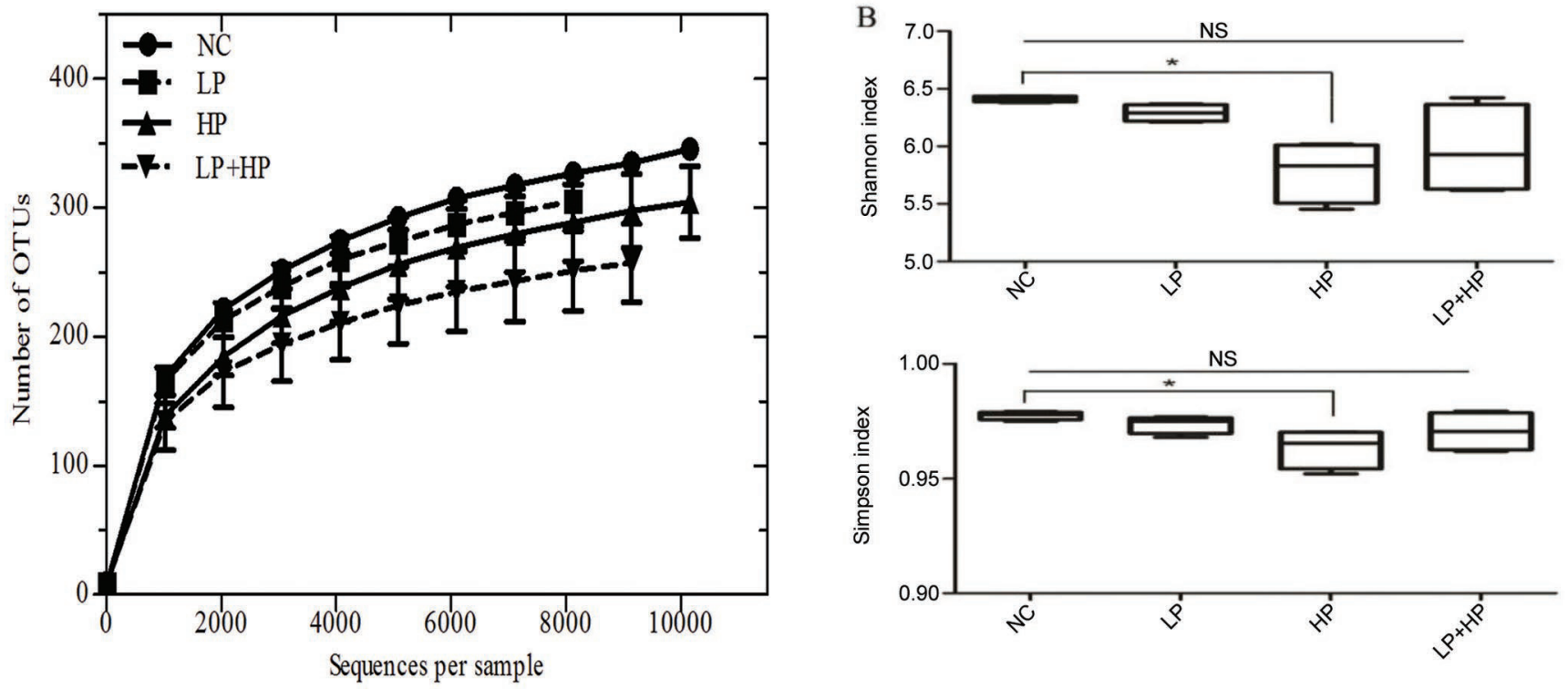

$\mathrm{C}$

\section{Sequencesper sample}
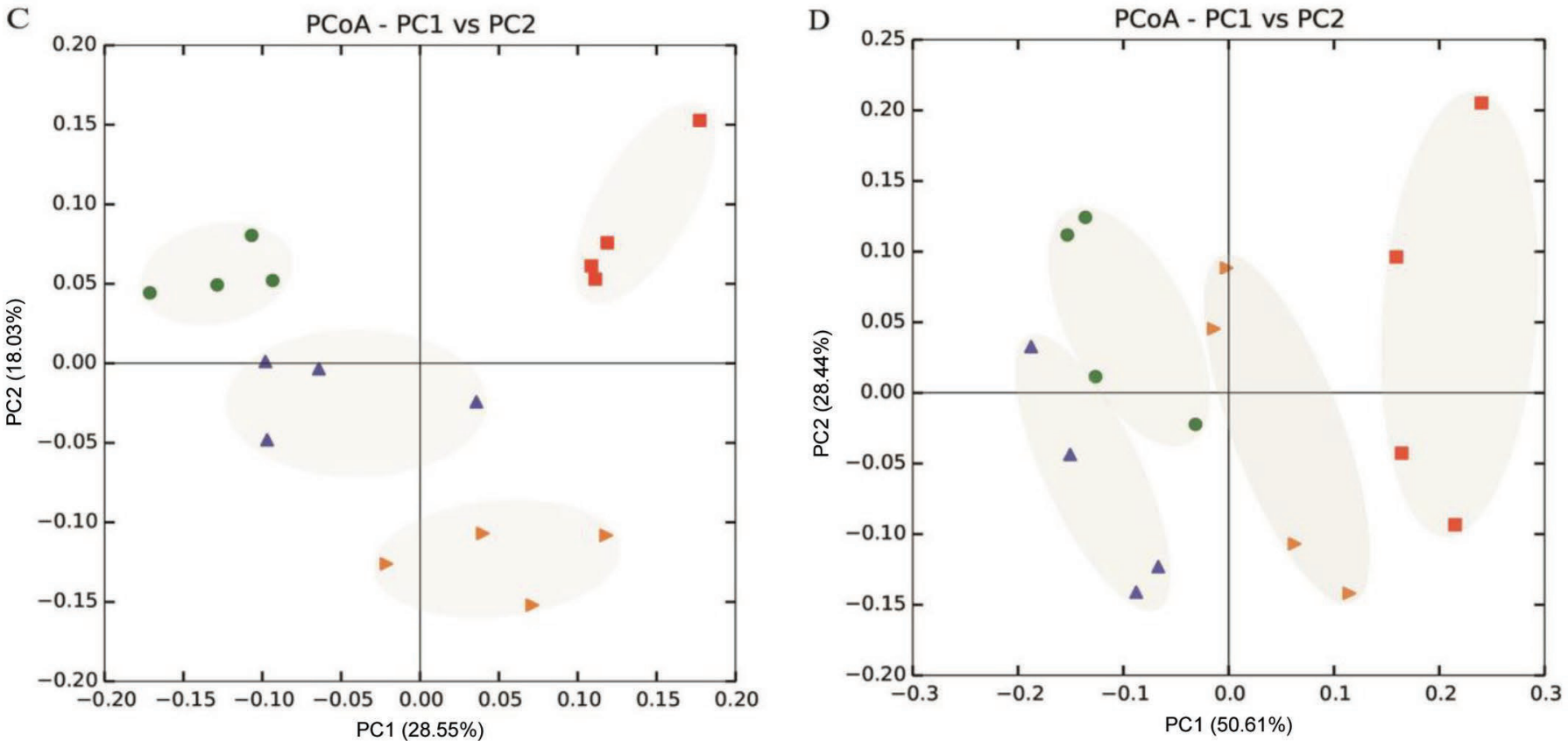

Figure 3. Bacterial community diversities and structures of the mice gastric microbiota in different treatments. (A) Rarefaction curves of operational taxonomic units (OTU) reached saturation, reflecting that samples had the most bacterial diversity within a sample. (B) Helicobacter pylori infection induced a decline in Shannon and Simpson index, and pretreatment with LP prevented this decline. ${ }^{*} P<0.05$ by ANOVA followed by Tukey post hoc test. Error bars represent mean \pm standard deviation. (C) Principal coordinate analysis (PCoA) showed an apparent clustering of the gastric microbiota of 4 groups based on unweighted Unifrac distance. (D) Principal coordinate analysis based on weighted Unifrac distance showed a robust clustering of the gastric microbiota of the 4 groups. Blue triangles represent groups of LP, green circles represent groups of $\mathrm{NC}$, pink triangles represent groups of $\mathrm{LP}+\mathrm{HP}$, and red squares represent groups of $\mathrm{HP}$. NC $=$ normal chow diet with $400 \mu \mathrm{L}$ of PBS (control); LP = normal chow diet with $400 \mu \mathrm{L}$ of viable cells of Lactobacillus plantarum ZDY $2013\left(\sim 10^{9} \mathrm{cfu} / \mathrm{mL}\right)$ suspended in PBS and administered by oral gavages; $\mathrm{HP}=$ normal chow diet with $400 \mu \mathrm{L}$ of PBS; LP+HP = normal chow diet with $400 \mu \mathrm{L}$ of viable cells of $L$. plantarum ZDY $2013\left(\sim 10^{9} \mathrm{cfu} / \mathrm{mL}\right)$ suspended in PBS and administered by oral gavages. Color version available online.

Ruminococcaceae, Coriobacteriaceae, Staphylococcaceae, and Bifidobacteriaceae) were much more abundant in the gastric microbiota of LP group, whereas at the genus level, 4 bacterial genera, including Rikenella, Staphylococcus, Bifidobacterium, and Corynebacterium, were observed. 


\section{DISCUSSION}

Lactobacillus plantarum ZDY 2013 was previously proven to be an ideal probiotic organism due to its high survival under acidic conditions and exhibition of acid tolerance response (Huang et al., 2015). In this study, L. plantarum ZDY 2013 was administered for its preventive effect against $H$. pylori infection. Our results demonstrated that pretreatment with L. plantarum ZDY 2013 can prevent gastric mucosal inflammation and gastric microbiota alteration induced by $H$. pylori infection.

For host immunity stimulation, H. pylori has been implicated in triggering the Th1/Th17 cell response in the gastric mucosa (Shi et al., 2010). In agreement with that, in this study we found an elevated level of inflammatory cytokine RNA for IL- $1 \beta$, IFN- $\gamma$, and IL- 17 at 6 wk postinfection, whereas in the $\mathrm{LP}+\mathrm{HP}$ group, an elevated mRNA level for IL- $1 \beta$ and IFN- $\gamma$ was inhibited, indicating that pretreatment with $L$. plantarum ZDY 2013 could prevent the Th1 cell response triggered by H. pylori infection. Numerous reports have mentioned the immune-modulatory properties of probiotics. However, pro- or anti-inflammation for the host immune system might occur on a case by case basis upon use of different probiotic strains (Turroni et al., 2014). Previous studies have reported an anti-inflammatory property of Lactobacillus plantarum in several animal disease models (Liu et al., 2011; Thiraworawong et al., 2014). Studies in the mouse colitis model demonstrated that an anti-inflammatory cytokine of IL-10 was upregulated by Lactobacillus plantarum supplementation (Duary et al., 2012); however, no significant increase in IL-10 was found in L. plantarum ZDY 2013 pretreated
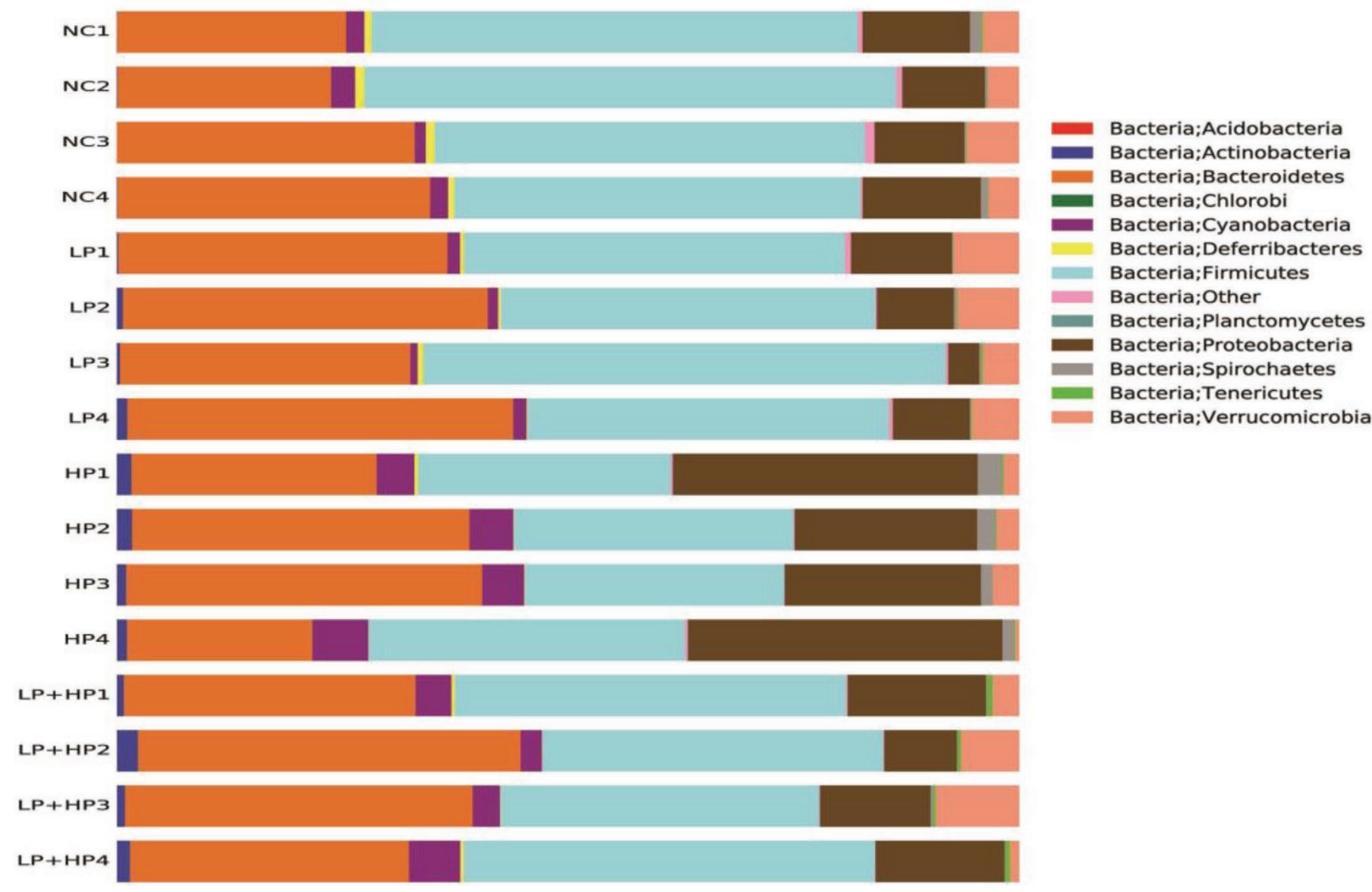

Figure 4. Composition of mice gastric bacterial community in different treatments. Each bar represents the relative abundance of operational taxonomic units (OTU) at the phylum level in the gastric microbiota within a sample. Firmicutes and Bacteroidetes represented on an average of as much as $75.6 \%$ of each sample's OTU. $\mathrm{NC}=$ normal chow diet with $400 \mu \mathrm{L}$ of PBS (control); LP = normal chow diet with $400 \mu \mathrm{L}$ of viable cells of Lactobacillus plantarum ZDY $2013\left(\sim 10^{9} \mathrm{cfu} / \mathrm{mL}\right)$ suspended in PBS and administered by oral gavages; HP = normal chow diet with $400 \mu \mathrm{L}$ of PBS; LP $+\mathrm{HP}=$ normal chow diet with $400 \mu \mathrm{L}$ of viable cells of $L$. plantarum ZDY $2013\left(\sim 10^{9}\right.$ cfu/mL) suspended in PBS and administered by oral gavages. 
mice, suggesting that other factors rather than IL-10 were involved in the anti-inflammatory activity of $L$. plantarum ZDY 2013.
Probiotics can affect the immune homeostasis by altering the microbial balance in the gastrointestinal tract and thus enhancing the immune system of the

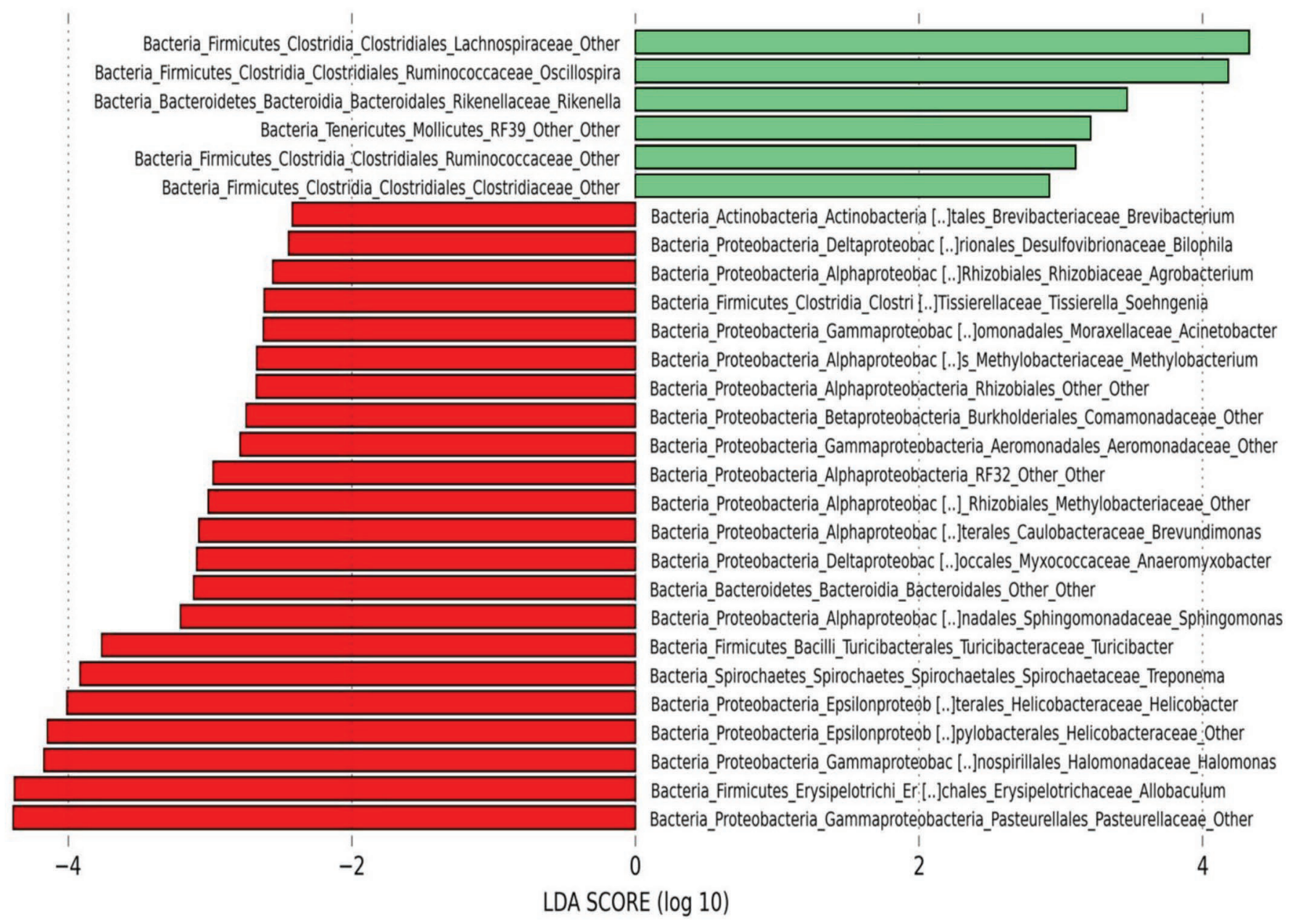

B

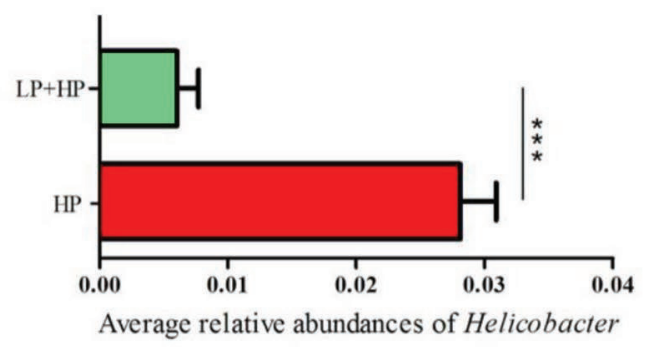

Figure 5. Difference in bacterial taxa between group of HP and LP+HP. (A) Linear discriminant analysis (LDA) coupled with effect size (LEfSe) showed that the bacterial taxa significantly differentiated between HP and LP + HP group. The HP group-enriched bacterial taxa are indicated with negative LDA score (red), and bacterial taxa enriched in LP $+\mathrm{HP}$ group are indicated with a positive LDA score (green). $P<$ 0.05 , LDA significant threshold $>2$. (B) Average relative abundances of Helicobacter in HP and LP $+\mathrm{HP}$. $* * * P<0.001$ by analysis of unpaired 2 -tailed Student's $t$-test. Error bars represent mean \pm standard deviation. $\mathrm{NC}=$ normal chow diet with $400 \mu \mathrm{L}$ of PBS (control); LP $=$ normal chow diet with $400 \mu \mathrm{L}$ of viable cells of Lactobacillus plantarum ZDY $2013\left(\sim 10^{9} \mathrm{cfu} / \mathrm{mL}\right)$ suspended in PBS and administered by oral gavages; $\mathrm{HP}=$ normal chow diet with $400 \mu \mathrm{L}$ of PBS; LP $+\mathrm{HP}=$ normal chow diet with $400 \mu \mathrm{L}$ of viable cells of L. plantarum ZDY $2013\left(\sim 10^{9} \mathrm{cfu} / \mathrm{mL}\right)$ suspended in PBS and administered by oral gavages. Color version available online. 
host (Clemente et al., 2012; Turroni et al., 2014). Due to the extreme acidic environment of the stomach, few probiotics can resist such conditions and remain alive. Upon the extreme gastric environment resistance of L. plantarum ZDY 2013, we examined the effect of its pretreatment on gastric microbiota post- $H$. pylori infection. In our study, H. pylori infection resulted in the decrease of $\alpha$ diversity accessed by Shannon index and Simpson index, L. plantarum ZDY 2013 pretreatment prevented that tendency to decrease. Furthermore, a significant gastric microbiota separation between NC and HP (Adonis test $<0.05$ ) was found according to PcoA analysis based on Unifrac distance, whereas nonsignificant gastric microbiota separation was found between $\mathrm{NC}$ and $\mathrm{LP}+\mathrm{HP}$ (Adonis test $>0.05$ ), indicating L. plantarum ZDY 2013 pretreatment corrected the alteration of microbiota induced by $H$. pylori. Selective modulation of the microbiota by using probiotics is a developing strategy for many diseases such as obesity (Delzenne et al., 2011), inflammatory bowel disease (Jones and Foxx-Orenstein, 2007), and liver disease (Xu et al., 2012). However, with regard to the beneficial effects of probiotics against $H$. pylori infection, the pro- posed mechanisms were limited to the biological properties of the strain itself, such as immune-modulatory (Yang and Sheu, 2012) and antimicrobial effects (Chen et al., 2012), due to previous bias that the normal stomach is sterile. Up to date, little was known about the niche of gastric flora, regardless of the change in gastric microbiota by intervention with lactic acid bacteria. Thus, to our knowledge, this is the first study to examine the effect of probiotics on gastric microbiota against $H$. pylori infection.

To further ascertain the bacterial taxa associated with H. pylori infection and L. plantarum ZDY 2013 pretreatment, LEfSe analysis was performed. We found that 22 bacterial taxa were more dominant in the HP group; most of them belonged to the Proteobacteria phylum, whereas in the LP group, 6 dominant phyla were present, mainly from Firmicutes and Bacteriodetes. The expansion of the Proteobacteria was strongly associated with several diseases because this phylum includes gram-negative bacteria, which includes a wide variety of pathogenic species, such as Escherichia, Salmonella, and Vibrio (Mukhopadhya et al., 2012). In this study, the inhibition of Proteobacteria in LP $+\mathrm{HP}$

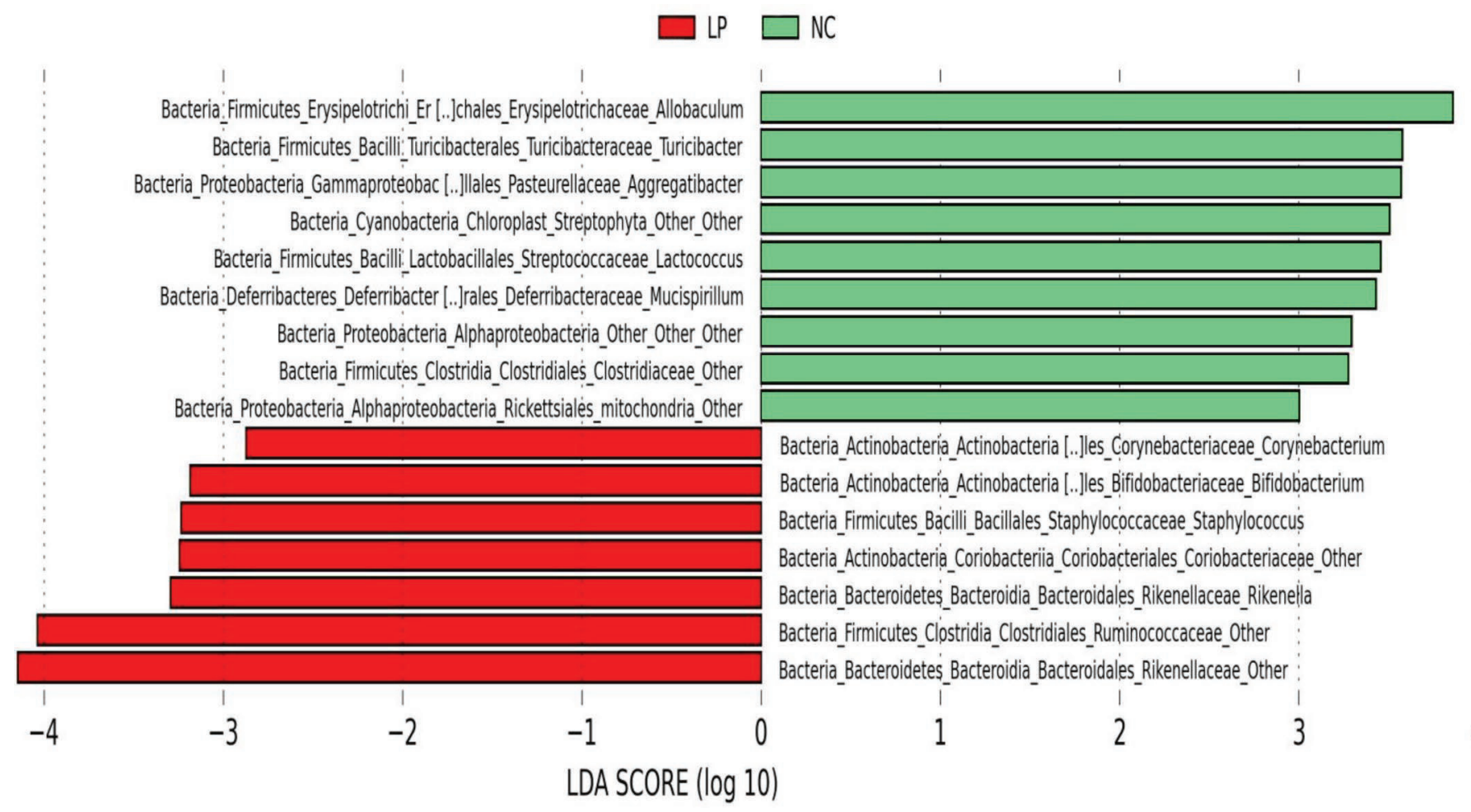

Figure 6. Difference in bacterial taxa between group of NC and LP. Linear discriminant analysis (LDA) coupled with effect size showed the significantly different bacterial taxa between NC and LP groups. The LP group-enriched bacterial taxa are indicated with negative LDA score (red), and NC group-enriched bacterial taxa with a positive LDA score (green). $P<0.05$, LDA significant threshold $>2$. NC $=$ normal chow diet with $400 \mu \mathrm{L}$ of PBS (control); LP = normal chow diet with $400 \mu \mathrm{L}$ of viable cells of Lactobacillus plantarum ZDY 2013 ( $\sim 10^{9}$ cfu/ $\mathrm{mL}$ ) suspended in PBS and administered by oral gavages; HP = normal chow diet with $400 \mu \mathrm{L}$ of PBS; LP $+\mathrm{HP}=$ normal chow diet with 400 $\mu \mathrm{L}$ of viable cells of L. plantarum ZDY $2013\left(\sim 10^{9} \mathrm{cfu} / \mathrm{mL}\right)$ suspended in PBS and administered by oral gavages. Color version available online. 
indicates that L. plantarum ZDY 2013 pretreatment successfully prevented the gastric microbiota alteration induced by $H$. pylori infection. Particularly, analysis of the abundance of bacterial genus of Helicobacter alone found a significant decrease in Helicobacter (from 0.0281 to 0.006 ), suggesting that colonization of Helicobacter was not totally prevented but suppressed by $L$. plantarum ZDY 2013 pretreatment.

For examination of the beneficial mechanism of probiotics on the host, ample evidence suggests that probiotics can inhibit potential pathogens directly through production of antimicrobial substance (Gotteland et al., 2006). Currently, other indirect benefits of probiotics administration including increasing the diversity or number of "good" bacteria, such as Bifidobacterium, Fecalibacterium, and Clostridium, and so on, have also been reported (Zhang et al., 2014). Thus, we are interested in the hypothesis that the pretreatment with L. plantarum ZDY 2013 might change the gastric microbiota, and in turn provide a preventive effect against H. pylori infection. According to the Adonis test, however, no significant microbiota alteration was found after $3 \mathrm{wk}$ of L. plantarum ZDY 2013 administration $(P>0.05)$. But through LEfSe analysis, we still found several bacterial taxa (e.g., Rikenella, Staphylococcus, Bifidobacterium) more abundant in L. plantarum ZDY 2013 treated mice when compared with the control group. These data suggest that the preventive effect of L. plantarum ZDY 2013 administration might occur through selective modulation of specific bacterial taxa in gastric microbiota rather than by changing the overall gastric microbiota.

In addition, except for its effect on stomach microbiota, the administration of L. plantarum ZDY 2013 and its effect on constitution of intestinal microbiota might be helpful against the infection of HP as well; therefore, as an another task, a systemic analysis of the gastrointestinal microbiota is worthy for exploring the mechanism of probiotic intervention against infection of H. pylori.

\section{CONCLUSIONS}

In summary, our results show that L. plantarum ZDY 2013 pretreatment posed a selective modulation in gastric microbiota, which may have contributed to an important preventive effect on $H$. pylori infection, including prevention in gastric mucosal inflammation and gastric microbiota alteration post- $H$. pylori infection. Our work suggests that targeting gastric microbiota through oral administration of probiotics might be an alternative strategy to prevent $H$. pylori infection.

\section{ACKNOWLEDGMENTS}

This study was sponsored by the National Natural Science Foundation of China (grant no. NSF 31360377), Academic and Technical Leaders Training Program for Major Subjects of Jiangxi Province (2009), and Ganpo Talent 555 Engineering Project of Jiangxi Province (China).

\section{REFERENCES}

Aviles-Jimenez, F., F. Vazquez-Jimenez, R. Medrano-Guzman, A. Mantilla, and J. Torres. 2014. Stomach microbiota composition varies between patients with non-atrophic gastritis and patients with intestinal type of gastric cancer. Sci Rep. 4:4202.

Bik, E. M., P. B. Eckburg, S. R. Gill, K. E. Nelson, E. A. Purdom, F. Francois, G. Perez-Perez, M. J. Blaser, and D. A. Relman. 2006. Molecular analysis of the bacterial microbiota in the human stomach. Proc. Natl. Acad. Sci. USA 103:732-737.

Caporaso, J. G., J. Kuczynski, J. Stombaugh, K. Bittinger, F. D. Bushman, E. K. Costello, N. Fierer, A. G. Pena, J. K. Goodrich, and J. I. Gordon. 2010. QIIME allows analysis of high-throughput community sequencing data. Nat. Methods 7:335-336.

Caporaso, J. G., C. L. Lauber, W. A. Walters, D. Berg-Lyons, J. Huntley, N. Fierer, S. M. Owens, J. Betley, L. Fraser, and M. Bauer. 2012. Ultra-high-throughput microbial community analysis on the Illumina HiSeq and MiSeq platforms. ISME J. 6:1621-1624.

Chen, X., X. M. Liu, F. Tian, Q. Zhang, H. P. Zhang, H. Zhang, and W. Chen. 2012. Antagonistic activities of lactobacilli against $\mathrm{He}$ licobacter pylori growth and infection in human gastric epithelial cells. J. Food Sci. 77:M9-M14.

Clemente, J. C., L. K. Ursell, L. W. Parfrey, and R. Knight. 2012 The impact of the gut microbiota on human health: An integrative view. Cell 148:1258-1270.

Delzenne, N. M., A. M. Neyrinck, F. Bäckhed, and P. D. Cani. 2011. Targeting gut microbiota in obesity: Effects of prebiotics and probiotics. Nat. Rev. Endocrinol. 7:639-646.

Duary, R. K., M. A. Bhausaheb, V. K. Batish, and S. Grover. 2012. Anti-inflammatory and immunomodulatory efficacy of indigenous probiotic Lactobacillus plantarum Lp91 in colitis mouse model. Mol. Biol. Rep. 39:4765-4775.

Gotteland, M., O. Brunser, and S. Cruchet. 2006. Systematic review: Are probiotics useful in controlling gastric colonization by Helicobacter pylori? Aliment. Pharmacol. Ther. 23:1077-1086.

Huang, R., X. Tao, C. Wan, S. Li, H. Xu, F. Xu, N. P. Shah, and H. Wei. 2015. In vitro probiotic characteristics of Lactobacillus plantarum ZDY 2013 and its modulatory effect on gut microbiota of mice. J. Dairy Sci. 98:5850-5861.

Joint FAO/WHO Working Group. 2002. Guidelines for the evaluation of probiotics in foods. Food and Agricultural Organization of the United Nations, Rome, Italy; World Health Organization, Geneva, Switzerland.

Jones, J. L., and A. E. Foxx-Orenstein. 2007. The role of probiotics in inflammatory bowel disease. Dig. Dis. Sci. 52:607-611.

Ley, R. E., F. Bäckhed, P. Turnbaugh, C. A. Lozupone, R. D. Knight, and J. I. Gordon. 2005. Obesity alters gut microbial ecology. Proc. Natl. Acad. Sci. USA 102:11070-11075.

Liu, Y.-W., Y.-W. Su, W.-K. Ong, T.-H. Cheng, and Y.-C. Tsai. 2011. Oral administration of Lactobacillus plantarum K68 ameliorates DSS-induced ulcerative colitis in BALB/c mice via the anti-inflammatory and immunomodulatory activities. Int. Immunopharmacol. 11:2159-2166.

Martin, M. E., and J. V. Solnick. 2014. The gastric microbial community, Helicobacter pylori colonization, and disease. Gut Microbes 5:345-350

McFarland, L. V., P. Malfertheiner, Y. Huang, and L. Wang. 2015. Meta-analysis of single strain probiotics for the eradication of $\mathrm{He}$ - 
licobacter pylori and prevention of adverse events. World J. MetaAnal. 3:97-117.

Mukhopadhya, I., R. Hansen, E. M. El-Omar, and G. L. Hold. 2012. IBD - What role do Proteobacteria play? Nat. Rev. Gastroenterol. Hepatol. 9:219-230

Oksanen, J., F. G. Blanchet, R. Kindt, P. Legendre, P. R. Minchin, R. O'Hara, G. L. Simpson, P. Solymos, M. Stevens, and H. Wagner. 2013. Package 'vegan'. R Package ver. 254:20-8.

Packey, C. D., and R. Sartor. 2008. Interplay of commensal and pathogenic bacteria, genetic mutations, and immunoregulatory defects in the pathogenesis of inflammatory bowel diseases. J. Intern. Med. 263:597-606

Pan, M., Q. Wu, X. Tao, C. Wan, N. P. Shah, and H. Wei. 2015. Fermentation of Allium chinense bulbs with Lactobacillus plantarum ZDY 2013 shows enhanced biofunctionalities, and nutritional and chemical properties. J. Food Sci. 80:M2272-M2278.

Patel, A., N. Shah, and J. Prajapati. 2014. Clinical application of probiotics in the treatment of Helicobacter pylori infection-A brief review. J. Microbiol. Immunol 47:429-437.

Polk, D. B., and R. M. Peek. 2010. Helicobacter pylori: Gastric cancer and beyond. Nat. Rev. Cancer 10:403-414.

Qin, N., F. Yang, A. Li, E. Prifti, Y. Chen, L. Shao, J. Guo, E. Le Chatelier, J. Yao, and L. Wu. 2014. Alterations of the human gut microbiome in liver cirrhosis. Nature 513:59-64.

Sakarya, S., and N. Gunay. 2014. Saccharomyces boulardii expresses neuraminidase activity selective for $\alpha 2$, 3-linked sialic acid that decreases Helicobacter pylori adhesion to host cells. APMIS 122:941950.

Salama, N. R., M. L. Hartung, and A. Müller. 2013. Life in the human stomach: Persistence strategies of the bacterial pathogen Helicobacter pylori. Nat. Rev. Microbiol. 11:385-399.

Segata, N., J. Izard, L. Waldron, D. Gevers, L. Miropolsky, W. S. Garrett, and C. Huttenhower. 2011. Metagenomic biomarker discovery and explanation. Genome Biol. 12:R60.

Sheh, A., and J. G. Fox. 2013. The role of the gastrointestinal microbiome in Helicobacter pylori pathogenesis. Gut Microbes 4:505-531.

Shi, Y., X.-F. Liu, Y. Zhuang, J.-Y. Zhang, T. Liu, Z. Yin, C. Wu, X.-H. Mao, K.-R. Jia, and F.-J. Wang. 2010. Helicobacter pyloriinduced Th17 responses modulate Th1 cell responses, benefit bacterial growth, and contribute to pathology in mice. J. Immunol. 184:5121-5129.

Thiraworawong, T., J. K. Spinler, D. Werawatganon, N. Klaikeaw, S. F. Venable, J. Versalovic, and S. Tumwasorn. 2014. Anti-in- flammatory properties of gastric-derived Lactobacillus plantarum XB7 in the context of Helicobacter pylori infection. Helicobacter 19:144-155.

Turroni, F., M. Ventura, L. F. Buttó, S. Duranti, P. W. O’Toole, M. O. C. Motherway, and D. van Sinderen. 2014. Molecular dialogue between the human gut microbiota and the host: A Lactobacillus and Bifidobacterium perspective. Cell. Mol. Life Sci. 71:183-203.

Wang, L.-L., X.-J. Yu, S.-H. Zhan, S.-J. Jia, Z.-B. Tian, and Q.-J. Dong. 2014. Participation of microbiota in the development of gastric cancer. World J. Gastroenterol. 20:4948-4952.

Weichert, S., H. Schroten, and R. Adam. 2012. The role of prebiotics and probiotics in prevention and treatment of childhood infectious diseases. Pediatr. Infect. Dis. J. 31:859-862.

Wroblewski, L. E., R. M. Peek, and K. T. Wilson. 2010. Helicobacter pylori and gastric cancer: Factors that modulate disease risk. Clin. Microbiol. Rev. 23:713-739.

Xu, J., F. Lian, L. Zhao, Y. Zhao, X. Chen, X. Zhang, Y. Guo, C. Zhang, Q. Zhou, and Z. Xue. 2015. Structural modulation of gut microbiota during alleviation of type 2 diabetes with a Chinese herbal formula. ISME J. 9:552-562.

Xu, R.-Y., Y.-p. Wan, Q.-Y. Fang, W. Lu, and W. Cai. 2012. Supplementation with probiotics modifies gut flora and attenuates liver fat accumulation in rat nonalcoholic fatty liver disease model. J. Clin. Biochem. Nutr. 50:72-77.

Yang, I., S. Nell, and S. Suerbaum. 2013. Survival in hostile territory: The microbiota of the stomach. FEMS Microbiol. Rev. 37:736-761.

Yang, L., H. Kuang, W. Zhang, Z. P. Aguilar, Y. Xiong, W. Lai, H. $\mathrm{Xu}$, and H. Wei. 2015. Size dependent biodistribution and toxicokinetics of iron oxide magnetic nanoparticles in mice. Nanoscale 7:625-636

Yang, Y. J., and B. S. Sheu. 2012. Probiotics-containing yogurts suppress Helicobacter pylori load and modify immune response and intestinal microbiota in the Helicobacter pylori-infected children. Helicobacter 17:297-304.

Zhang, J., L. Wang, Z. Guo, Z. Sun, Q. Gesudu, L. Kwok, and H. Zhang. 2014. 454 pyrosequencing reveals changes in the faecal microbiota of adults consuming Lactobacillus casei Zhang. FEMS Microbiol. Ecol. 88:612-622.

Zhang, L., J. Yu, C. Wong, T. Ling, Z. Li, K. Chan, S. Ren, J. Shen, R. Chan, and C. Lee. 2013. Cathelicidin protects against Helicobacter pylori colonization and the associated gastritis in mice. Gene Ther. $20: 751-760$ 